\title{
A new gold (old) standard for nose reconstruction: The one-stage midline forehead flap - a 13-year retrospective review of results using the central vein and inverted-kite pedicle base modification
}

\author{
W G Kleintjes, MMed (Plast), FC (Plast Surg) SA, PhD \\ Western Cape Provincial Tertiary Adult Burn Unit, Division of Surgery, Tygerberg Hospital and Stellenbosch University, South Africa
}

Corresponding author: W G Kleintjes (waynekleintjes@yahoo.com)

\begin{abstract}
Background. A detailed study of the blood supply of the forehead has led to precise landmarks for midline forehead flap planning, similar to the old Indian rhinoplasty landmarks described by Daver and Antia. Further investigation into pedicle rotations has shown that the best technique for one-stage pedicle inset was to use an inverted-kite pedicle base modification of the midline forehead flap.

Objective. To carry out a retrospective review of all the cases using the one-stage inverted-kite midline forehead flap (OSIKMFF) operated on by the author between January 2006 and September 2018.

Methods. The retrospective review gathered information on patients' age, pathology, flap design and complications.

Results. Twenty patients were included in the study. Most data for 2008 were, unfortunately, lost, but patients whose data were retrievable were included. The average age of the patients was 57 years (range 21 - 84). The male-to-female ratio was 1:1 (10 v. 10). The most common pathology was basal cell carcinoma, which was present in 18/20 (90\%) cases, and the remaining 2 (10\%) patients had melanoma. Only in two (10\%) cases was tissue expansion used before the OSIKMFF. All the flaps survived, and the blood supply was very robust. Complications were minor.

Conclusion. The one-stage forehead flap using the inverted-kite pedicle base modification is a useful technique, based on meticulously identified safe landmarks, the robust blood supply, meticulous cadaver dissections and clinical experience over many years.
\end{abstract}

SAfr J Plast Reconstr Aesthet Surg Burns 2018;1(1):10-14. DOI: 10.7196/SAJPRASB.2018.v1i1.4

Forehead flaps are well known for their reliable blood supply, hence the successful use of this flap over thousands of years. ${ }^{[1,2]}$ A detailed study of the blood supply of the forehead has led to the identification of precise landmarks for midline forehead flap planning, similar to the old Indian rhinoplasty landmarks described by Daver and Antia. ${ }^{\left[{ }^{[3]}\right.}$ Further investigation into pedicle rotations has shown that the best technique for one-stage pedicle inset was to use an inverted-kite pedicle base modification of the midline forehead flap. ${ }^{[4]}$ Other authors have described their positive experiences with the one-stage forehead flap, but despite this, the paramedian forehead flap became the gold standard flap in the West. ${ }^{[5-10]}$

The correct application of the landmarks and pedicle base modification allows for one-stage pedicle inset of a reliable robust flap, and almost no need for pedicle separation at a second stage. ${ }^{[4]}$ This depends on the reconstructive demands of the defect. In some cases, only pedicle base refinements or minor debulking may be necessary. Some cases may require complete flap elevation, in which case the blood supply and sensation can be safely maintained, which is not practical with a paramedian forehead flap once the pedicle has been separated. The one-stage forehead flap may be highly advantageous for surgeons doing missionary surgery where surgeon follow-up is suboptimal, and onestage primary surgery with minimal secondary operations is ideal. It is also a useful procedure in elderly patients, for whom ultimate aesthetic outcomes may be less important. Using fewer stages means that there is less cosmetic refinement, but using the one-stage inset technique does not mean that further stages for refinement cannot be done. In the author's experience, the paramedian forehead flap has become as obsolete as a cross-leg flap.

\section{Methods}

A retrospective review was carried out of all the cases operated on from January 2006 until September 2018 by the author. The technique used for midline forehead flap planning is as follows: any scars or injuries to the arterial pedicles must be excluded by clinical examination of the facial skin overlying the source arteries, from the facial artery to the angular artery, the dorsal nasal artery, the central artery, the paramedian and the supratrochlear artery communicating branch area in the medial canthal region. ${ }^{[2]}$ Next, a central vein of the forehead should be found. This vein is more prominent when patients frown, become emotional, or valsalva, or when they are in a prone position. The vein is a cutaneous landmark of the subcutaneous arterial patterns. ${ }^{[3]}$ When present, the central vein is then used as the central axis for the flap. Since the central arteries are tightly woven around the central vein and have interarterial communications, it is possible to design a narrow pedicle of about $5-10 \mathrm{~mm}$ in width ${ }^{[4]}$ As the central vein drains into either left or right orbits in the supramedial canthal region (the frontonasal angle), the pedicle base will turn in that direction. A rare central arterial and venous variation is that the vein may be exactly in the midline, and fork at the glabella to drain into the medial canthal areas. ${ }^{[4]}$ This vein has been named a median vein, and has a corresponding 
median artery ${ }^{[3]}$ The limits of the medial pedicle margin are the midline, the medial canthal horizontal line inferiorly to a point $5 \mathrm{~mm}$ medial to the medial canthal vertical line. The lateral pedicle margin should also not be closer than $5 \mathrm{~mm}$ to the medial canthal vertical line at the pedicle base over the medial orbital rim (Fig. 1). In cases where a central vein cannot be seen or found, the pedicle is designed according to the landmarks given (Fig. 1).

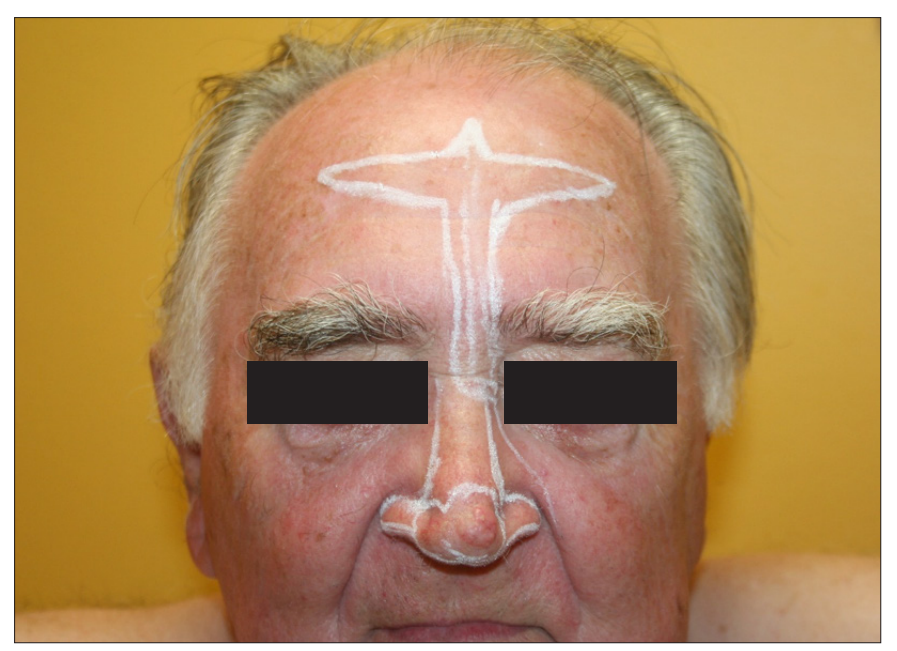

Fig. 1. Safe landmarks for midline forehead flap design are shown, with or without a central vein as the flap axis.

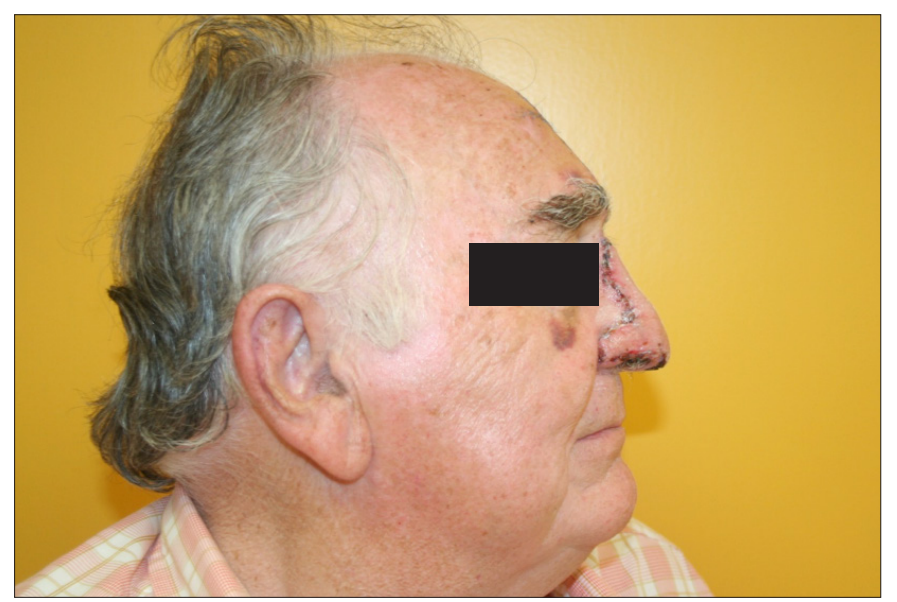

Fig. 2. Profile after flap inset has no humps, and patient can safely wear glasses.

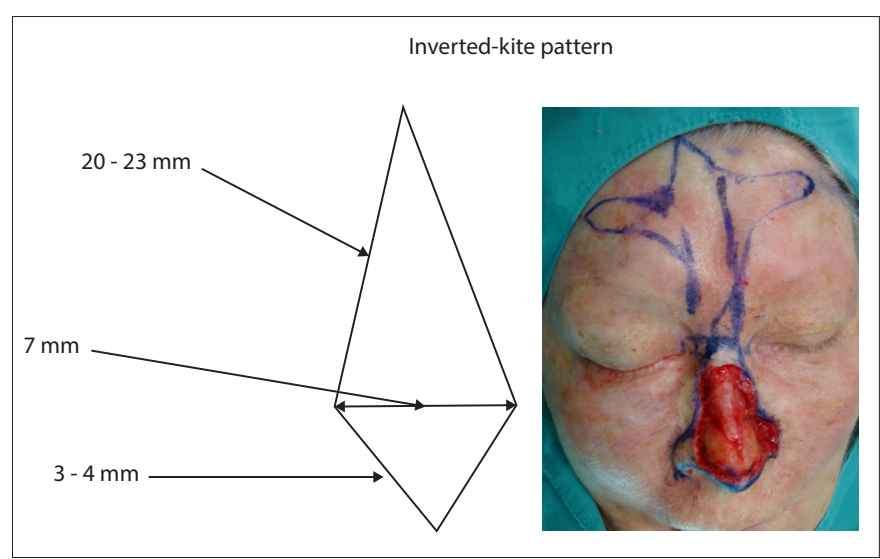

Fig. 3. Inverted-kite pedicle design for one-stage flap inset.
The best design of the pedicle to achieve a one-stage inset was studied in five cadavers. ${ }^{[4]}$ The inverted-kite design gave the best profile result for pedicle inset for a one-stage midline forehead flap (Fig. 2). The dimensions of the inverted-kite skin excision at the pedicle base are approximately $20 \mathrm{~mm}$ superiorly $\times 7 \mathrm{~mm}$ wide $\times 3 \mathrm{~mm}$ inferiorly (Fig. 3). Only epidermis and dermis with minimal subdermal fat is excised when cutting and removing the inverted-kite pattern at the pedicle base. Adjacent lateral nasal wall skin is dissected loose with scissors. A further requirement for the one-stage inlay is that the dorsal nasal skin must be removed, to make place for the flap and pedicle.

\section{Results}

A total of 20 patients were included. The demographic data of the patients are shown in Table 1. Most data for 2008 were, unfortunately, lost. All patients whose data were retrievable were included.

The average age of the patients was 57 years (range 21 - 84 years). The male-to-female ratio was 1:1 (10 v. 10). The most common pathology was basal cell carcinoma, which was present in 18/20 (90\%) cases, and the remaining two (10\%) patients had melanoma. Only in two (10\%) cases was tissue expansion used before the one-stage inverted-kite midline forehead flap (OSIKMFF).

All the flaps survived, and the blood supply was very robust (see Fig. 4). There were some relatively minor complications, such as minor dehiscence of an ala and postoperative swelling (2/20; $10 \%)$, and cosmetic revisions for slight bulging at the pedicle inset (2/20; 10\%). Minor pedicle base modifications were made in four cases, 2 weeks postoperatively, for cosmetic purposes, to perfect the results (4/20; 20\%). One patient had partial necrosis of the left ala after sleeping with the ala on his arm on day 2 postoperatively $(1 / 20 ; 5 \%)$.

\section{Discussion}

The routine success of the OSIKMFF, despite not using a Doppler for pedicle identification, and even in cases without a visible central vein as pedicle axis landmark, can be ascribed to accurate landmarks for flap planning based on the underlying vascular anatomy (examples of results are shown in Fig. 5). The midline forehead flap was previously considered an axial flap, based on the supratrochlear artery, and some reports identified branches from the angular artery. ${ }^{[11-14]}$ The more accurate identification of the axial arteries of the midline forehead flap was demonstrated to be the central

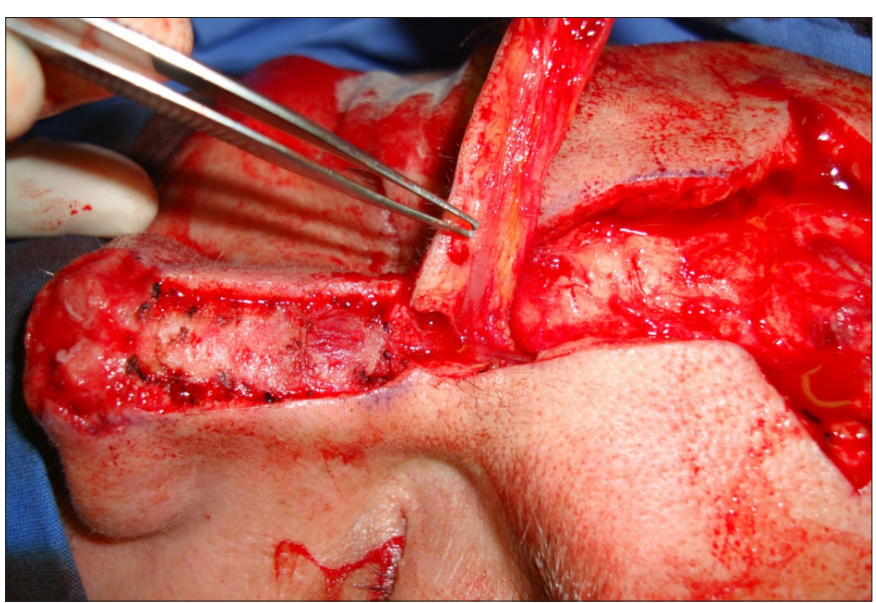

Fig. 4. Flap elevation with intact neurovascular bundle shown. 
Table 1. Demographic data of patients who had reconstruction using forehead flap technique (2006 - 2015)

\begin{tabular}{llllll}
\hline Patient & Year & Age & Sex & Pathology & Flap \\
\hline 1 & 2006 & 75 & $\mathrm{M}$ & Melanoma lower lid & MFF, CV \\
2 & 2006 & 84 & $\mathrm{M}$ & BCC nose and cheek & MFF, CV \\
3 & 2006 & 64 & $\mathrm{M}$ & BCC nose & MFF, CV \\
4 & 2007 & 43 & $\mathrm{~F}$ & BCC nose dorsum & OSIKMFF \\
5 & 2007 & 21 & $\mathrm{~F}$ & Burned nose & TE and OSIKMFF \\
6 & 2007 & 84 & $\mathrm{M}$ & BCC nose & OSIKMFF \\
7 & 2007 & 67 & $\mathrm{~F}$ & BCC nose & OSIKMFF \\
8 & 2008 & 83 & $\mathrm{~F}$ & BCC nose & OSIKMFF \\
9 & 2009 & 45 & $\mathrm{~F}$ & BCC lateral nose; cheek & OSIKMFF \\
10 & 2009 & 75 & $\mathrm{M}$ & BCC nose & OSIKMFF \\
11 & 2010 & 75 & $\mathrm{~F}$ & BCC nose & OSIKMFF \\
12 & 2011 & 30 & $\mathrm{M}$ & Dog bite nose & OSIKMFF \\
13 & 2011 & 26 & $\mathrm{~F}$ & BCC canthus and nose & OSIKMFF \\
14 & 2011 & 80 & $\mathrm{M}$ & Recurrent BCC nose & OSIKMFF \\
15 & 2012 & 51 & $\mathrm{~F}$ & BCC nose & OSIKMFF \\
16 & 2012 & 67 & $\mathrm{M}$ & Necrotising fasciitis & TE and OSIKMFF \\
17 & 2012 & 58 & $\mathrm{~F}$ & BCC nose & OSIKMFF \\
18 & 2015 & 82 & $\mathrm{~F}$ & Recurrent BCC & OSIKMFF \\
19 & 2015 & 53 & $\mathrm{M}$ & BCC nose & OSIKMFF \\
20 & 2015 & 75 & BCC nose & OSIKMFF
\end{tabular}

or paracentral arteries, originating from the dorsal nasal artery or from the angular artery. ${ }^{[4]}$ The use of the forehead central vein as a landmark for flap planning was proposed based on the close association of the central vein to the central artery and paracentral artery, as found in multiple cadaver dissections. ${ }^{[4]}$ It was shown that the central vein is not associated with the supratrochlear arteries. New terminology for the arteries associated with the vein was therefore introduced. ${ }^{[4]}$

There is no published randomised blinded prospective clinical study to compare the inclusion of the vein with a control where the vein is not included. Since the patterns and presence of the vein vary, the inclusion of the vein in the flap is not guaranteed by using the safe landmarks for flap design given, but the arteries are included. ${ }^{[4]}$ There is more variation of the supratrochlear artery in relation to unilateral absence in the hemiforehead, in comparison with the central artery ${ }^{[3]}$ This therefore indicates that the central arterial supply to the forehead is more consistent than the paramedian supply from the supratrochlear artery. Variations of the central vein have been described, and it has been shown that they may be present bilaterally to the midline, and in rare cases, a singular absolute median vein (and corresponding median artery) may be present, dividing at the nasal glabella area to drain bilaterally into the medial canthal areas. ${ }^{[4]}$ The vein is more commonly either to the left or right of the midline. ${ }^{[4]}$ The benefit of using the central vein, then, is that it is a macroscopic cutaneous landmark of the underlying arterial pattern, and thus makes the selection of the site and drawing of the pedicle easier. Secondly, and theoretically, it is always safer to include a vein with an artery in a flap, since flaps are more sensitive to venous occlusion than arterial occlusion. ${ }^{[15]}$ When the flap is planned according to the landmarks, and without a central vein identified, the inclusion of a vein is not guaranteed. A supratrochlear vein is not as common as a central vein, and therefore it cannot be used as a cutaneous landmark for the flap axis of a paramedian forehead flap as reliably as the midline forehead flap and central vein. ${ }^{[4]}$
We had a very low necrosis rate in our patients, which is in keeping with the literature. Historically, the midline forehead flap has been shown to be a robust flap, yet not without occasional partial flap necrosis. The application of the knowledge of the location and variation of the arterial pattern and central vein, theoretically and, as shown by this study, practically, can contribute to even safer and more robust pedicles. The low pedicle base position makes a long pedicle, as required with a paramedian forehead flap, unnecessary. The narrow pedicle allows for greater manoeuvring of the flap. The tortuous bend (or 'genu') of the supratrochlear artery as it passes through the frontalis muscle at, or superior to, the brow level may be compromised by rotation of a narrow pedicle in a paramedian forehead flap. ${ }^{[4]}$ The midline forehead flap provides more pedicle length owing to a shorter distance from the rotation point to the defect. It is the best choice in a short forehead, where, if one had to use a paramedian forehead flap, adjusted techniques such as oblique flap designs or tissue expansion might be considered in certain cases.

Another advantage of the midline over the paramedian forehead flap is that there is no pedicle (although only temporary) over the medial eye, obstructing vision, and no raw wound (especially compared to one-stage flap inlay), with its associated discomfort to the patient. In addition, a skin graft is not used at the pedicle base with the one-stage flap inlay.

The secondary modifications were for excision of excess skin, to obtain a smooth dorsal nose transition between flap and adjacent skin, or for minor bulges at the pedicle base due to flap rotation. The vascular pedicle of the flap is kept intact during the secondary revision. It is possible to elevate the skin over the pedicle base without damaging the pedicle. Most patients presenting with nasal skin cancers are elderly, and wear glasses, and the design of the one-stage inset allows patients to wear their glasses almost immediately postoperatively, as this does not compromise flap blood supply. The pressure points of spectacles, when worn, are usually laterally medial and inferior to the pedicle blood supply. Therefore, 
although there is a degree of compression at the base of the nose by the glasses, it has not led to any case of partial or total necrosis where the central vein planning was used. Some case results are shown in Figs 5A - 5D.

The paramedian forehead flap always requires a second stage, but the one-stage midline forehead flap does not.

The one-stage design allows minima secondary pedicle modification, the maintenance of the blood supply during secondary flap elevation, and wearing of glasses postoperatively.

Unlike in the technique used by Park, ${ }^{[8]}$ there is no need to resect any muscles, such as the procerus.

One sacrifice required in a one-stage inlay is the fact that the dorsal nose skin has to be removed. Although sacrificing normal tissue may seem a disadvantage, it is cosmetically beneficial, allowing blending of the cosmetic subunits of the nose. The dorsal nasal skin in most such patients has multiple areas of solar keratosis or solar damage, and therefore this might in general be good for the patient in the long run.

The benefits of the one-stage midline forehead flap using the central vein in comparison to the paramedian forehead flap are summarised in Table 2.

The only disadvantage of a one-stage forehead flap is that if further improvement of the cosmetic result is needed, then more stages will be required. The main benefit that makes the method stand out above the paramedian forehead flap, in this instance, is that the pedicle can be kept intact with any future stage refinements.

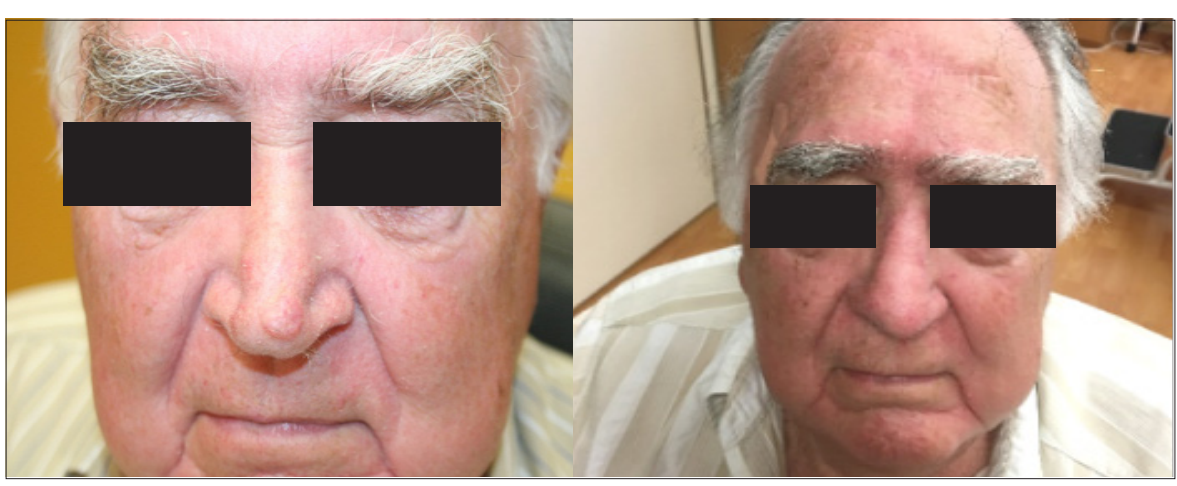

Figs 5 A and B. Patient 1, before and after OSIKMFF surgery.

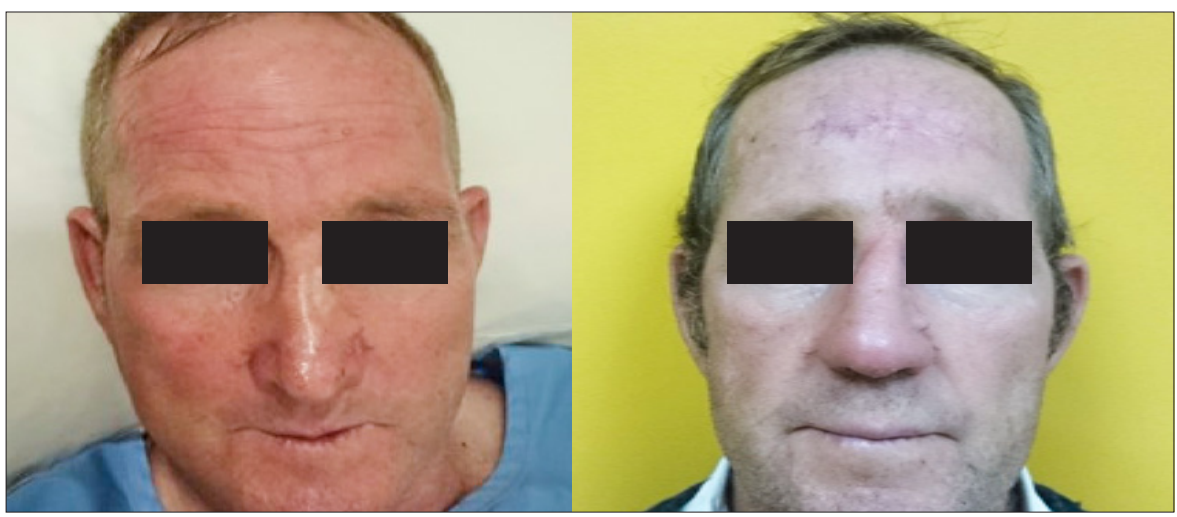

Figs 5C and D. Patient 2, before and after OSIKMFF surgery.
In the comparison of the advantages and disadvantages of the paramedian forehead flap v. the OSIKMFF, it is the latter that clearly results in less patient morbidity, and has greater advantages. With the superior advantages of

Table 2. Feature comparison of OSIKMFF v. PMFF use

\begin{tabular}{lll}
\hline Factor & OSIKMFF & PMFF \\
\hline Axial flap & Central; paracentral arteries & Supratrochlear artery \\
Cutaneous venous landmark & Often visible central vein & Seldom visible supratrochlear vein \\
Safe anatomic pedicle landmarks & Yes; minimal arterial variation (3\%) & Arterial variations and absence common (>60\%) \\
Inclusion of visible vein & Often & May require wider base to include vein \\
Narrow pedicle & Safe & May compromise flap \\
Pedicle length & Long possible; short forehead less problematic & Restricted; requires longer pedicle \\
Visual obstruction by pedicle & None & Common \\
Raw pedicle wound & None & Common \\
Second stage required & Seldom & Always \\
Pedicle flow cut at second stage & No; arterial and venous flow can be maintained & Yes \\
Secondary revision & Minor & Major compared with OIKMFF \\
Postoperative wearing of spectacles & No problem (beneficial to older patients) & Normal wear not possible \\
Sacrifice normal nose skin & The rule & Not the rule \\
Aesthetic subunits & Total resurfacing common & Not common \\
One stage & Always possible & Not recommended \\
Sensation in flap maintained & Yes & Seldom \\
Skin graft of pedicle & Never necessary & Necessary \\
Cost-effective & Yes & No \\
Early acceptable result & Yes & No \\
OsIKMFF = one-stage inverted-kite midline forehead flap; PMFF= paramedian forehead flap. &
\end{tabular}


the OSIKMFF, the history of robustness spanning more than 2500 years and the current detailed anatomic knowledge of the area, opting not to use the flap, and to rather use a paramedian forehead flap as a first choice, could be considered unethical. Patients should give full informed consent for an operation, and the onus of this ethical responsibility is on the treating surgeon. It is not inconceivable that patients who are well informed will choose the flap option with the best advantages and the least morbidity. We would do well to remember what our primary responsibility to our patients is in the Hippocratic Oath: primum non nocere - first do no harm. ${ }^{[16]}$

\section{Conclusion}

The one-stage forehead flap using the inverted-kite pedicle base modification is a useful technique, based on meticulously identified safe landmarks, robust blood supply, meticulous cadaver dissections and clinical experience over many years. It can also be of benefit in situations where patient follow-up is difficult, or in missionary surgery, where regular followup is not possible. The planning of the flap is made easier by identifying the central vein. Knowledge of the arterial and venous variations of the central forehead is essential, to increase one's understanding of potential variations in blood supply, and subsequent technical adjustments for flap refinements and safety. Based on the current evidence, and the experiences of many surgeons over 2500 years, the paramedian forehead flap should be reserved for cases where the blood supply of the central forehead is compromised and the midline forehead flap with one-stage inverted-kite inset should be the gold 'old' standard.
Acknowledgements. I would like to thank Prof. D F du Toit, Mr Reggie Williams, Mr Johannes Matthysen, Dr Marcel Geldenhuys, and students Guido Dees, Johannes Wimmer and Lynn Sampson, who helped me with the early research in 2004 and 2005.

Author contributions. Sole author.

Funding. None.

Conflicts of interest. None.

1. Eisenberg I. History of medicine. A history of rhinoplasty. S Afr Med J 1982;62(9):286-292.

2. Lascaratos J, Cohen M, Dionyssios V. Plastic surgery of the face in the fourth century. Plast Reconstr Surg 1998;102(4):1274-1280

3. Daver BM, Antia NH. Large Indian forehead rhinoplasty. Plast Reconstr Surg 1975:55(3): 360-362.

4. Kleintjes WG. Forehead anatomy: Arterial variations and venous link of the midline forehead flap. J Plast Reconst Aesthet Surg 2007; 60(6):593-606. https://doi.org/10.1016/j.bjps.2006.12.006

5. Tian BS. One-stage total rhinoplasty using a mid-forehead island flap: Report of 36 cases. Zhonghua Wai Ke Za Zhi Tian BS. One-stage

6. Xu Z. One-stage reconstruction following resection of oropharyngeal carcinoma. Zhonghua Er Bi Yan Hou Ke Z Zhi 1991;26(6):330-331.

7. Piza-Katzer H, Rath T, Dittrich P. One-stage nose reconstruction using a forehead island flap. HNO 1991;39(1):23-26. 8. Park SS. The single-stage forehead flap in nasal reconstruction: An alternative with advantages. Arch Facial Plast Surg 2002; $4(1): 32-36$.

9. Winslow CP, Cook TA, Burke A, et al. Total nasal reconstruction: Utility of the free radial facial flap. Arch Plast Surg 2003:5(2):159-163.

10. Rohrich RJ, Griffn JR, Ansari M, et al. Nasal reconstruction: Beyond aesthetic subunits: A 15-year review of 1334 cases Plast Reconstr Surg 2004;114(6):1405-1406.

11. Millard DR Jr. Reconstructive rhinoplasty of the lower two-thirds of the nose. Plast Reconstr Surg 1976:57(6):722-728 12. Cormack GC, Lamberty BGH, eds. The Arterial Anatomy of Skin Flaps. 2nd ed. Edinburgh: Livingstone, 1994:443-454

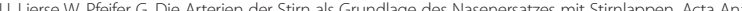
1980;107:18-25.

14. Shumrick KA, Smith TL. The anatomic basis for the design of forehead flaps in nasal reconstruction. 1992:118:373379.

15. Utley DS, Koch RJ, Good RL. The failing flap in plastic and reconstructive surgery: Role of the medicinal leech Laryngoscope 1998;108(8):1129-1135.

16. Body RI, Foex B. On the philosophy of diagnosis: Is doing more good than harm better than 'primum non nocere'? Emerg Med J 2009;26(4):238-240. https://doi.org/10.1136/emj.2008.064303

Accepted 8 November 2018 